Claude-Alain Faure, Université de Lausanne, Section de mathématiques, CH-1015 Lausanne-Dorigny, Switzerland

\title{
A DESCRIPTIVE DEFINITION OF THE KH-STIELTJES INTEGRAL ${ }^{\dagger}$
}

\begin{abstract}
This paper gives a descriptive definition of Stieltjes integrals (on a compact interval of the real line) in the frame of Kurzweil-Henstock integration. Five conditions characterize the functions that are an indefinite integral with respect to some continuous function of generalized bounded variation.
\end{abstract}

\section{Introduction}

A descriptive definition of the Kurzweil-Henstock integral, involving differentiability almost everywhere together with some null condition, is known since a few years (cf. for instance [3]). A more complete fundamental theorem was given by W. B. Jurkat and R. W. Knizia for the multidimensional weak integral in [4] and [5], where these authors introduced a useful and natural outer measure associated to any (interval) function.

In a preceding paper [1], I gave such a fundamental theorem for the multidimensional integrals of J. Mawhin [6] and W. F. Pfeffer [8]. In the present one, I propose a similar theorem for the Kurzweil-Henstock-Stieltjes integral on a compact interval $[a, b] \subseteq \mathbb{R}$. Five equivalent conditions thus characterize the functions $F:[a, b] \rightarrow \mathbb{R}$ which are an indefinite integral of some function $f:[a, b] \rightarrow \mathbb{R}$ relatively to $U:[a, b] \rightarrow \mathbb{R}$, cf. Theorem 4.7 and Corollary 5.6. The function $U$ is assumed to be continuous and $\mathrm{VBG}^{\circ}$ (equivalently, $\mathrm{VBG}_{*}$ in the sense of Saks [9]).

\footnotetext{
Key Words: gauge integral, Stieltjes sums, fundamental theorem of calculus

Mathematical Reviews subject classification: Primary 26A39, 26A42; Secondary 26A45

Received by the editors September 15, 1994

${ }^{\dagger}$ The Managing Editors apologize to the author for the delay in publication of this article.

* This work was done at the Catholic University of Louvain (Belgium) and it was supported by a grant from the Swiss National Science Foundation.
} 
Two difficulties arise in comparison with the non-Stieltjes case. First, the use of $\mathrm{VBG}^{\circ}$ functions requires technical adjustments in many proofs (often along the same lines). Then, especially, a strong theorem on relative differentiation is needed, cf. Theorem 3.2 (and [2] for a more general version).

At the end of the paper, as an application of the fundamental theorem, a substitution theorem is given for the Kurzweil-Henstock integral, which uses a measurable and bounded function $f$. Such a theorem is well-known for the Lebesgue integral, but I have not found any reference for the KH-integral.

\section{Preliminaries}

Definition 2.1. A system $S$ on a set $A \subseteq[a, b]$ is given by a finite family of intervals $a \leq a_{1}<b_{1} \leq \ldots \leq a_{r}<b_{r} \leq b$ together with a family of associated points $x_{i} \in\left[a_{i}, b_{i}\right] \cap A$. Now let $\delta: A \rightarrow \mathbb{R}_{+}$be any gauge on the set $A$. One says that the system $S$ is $\delta$-fine if $\left[a_{i}, b_{i}\right] \subseteq\left(x_{i}-\delta\left(x_{i}\right), x_{i}+\delta\left(x_{i}\right)\right)$ for every $i=1, \ldots, r$. We denote by $\mathcal{S}(A, \delta)$ the set of all $\delta$-fine systems $S$ on $A$.

Definition 2.2. A division of the interval $[a, b]$ is a system $D$ on $[a, b]$ which satisfies $b_{i}=a_{i+1}$ for every $i=0, \ldots, r$ (where $b_{0}=a$ and $a_{r+1}=b$ ). Given two functions $f, U:[a, b] \rightarrow \mathbb{R}$ one can form the Riemann-Stieltjes sum

$$
S(f, U, D)=\sum_{i=1}^{r} f\left(x_{i}\right)\left(U\left(b_{i}\right)-U\left(a_{i}\right)\right) .
$$

Then one says that the function $f$ is integrable relatively to the function $U$, or shortly that $f$ is $U$-integrable, if there exists a number $I \in \mathbb{R}$ such that for any $\varepsilon>0$ there exists a gauge $\delta:[a, b] \rightarrow \mathbb{R}_{+}$with the property

$$
|S(f, U, D)-I|<\varepsilon \text { for every } \delta \text {-fine division } D \text { of }[a, b] .
$$

The integral $I \in \mathbb{R}$ is clearly unique, and denoted by $\int_{a}^{b} f \mathrm{~d} U$. The following propositions 2.3 and 2.4 are well-known properties of the integral.

Proposition 2.3. Let $f, U:[a, b] \rightarrow \mathbb{R}$ and $a<c<b$. Then $f$ is integrable relatively to the function $U$ on the interval $[a, b]$ if and only if both integrals $\int_{a}^{c} f \mathrm{~d} U$ and $\int_{c}^{b} f \mathrm{~d} U$ exist. And one has $\int_{a}^{b} f \mathrm{~d} U=\int_{a}^{c} f \mathrm{~d} U+\int_{c}^{b} f \mathrm{~d} U$.

Proposition 2.4. Saks-Henstock Lemma Let $f:[a, b] \rightarrow \mathbb{R}$ be integrable relatively to the function $U:[a, b] \rightarrow \mathbb{R}$. We suppose given a gauge $\delta$ on the interval $[a, b]$ such that $\left|S(f, U, D)-\int_{a}^{b} f \mathrm{~d} U\right|<\varepsilon$ for every $\delta$-fine division $D$ of $[a, b]$. Then for any $\delta$-fine system $S$ one has the following inequalities:

1) $\left|\sum_{i=1}^{r}\left\{f\left(x_{i}\right)\left(U\left(b_{i}\right)-U\left(a_{i}\right)\right)-\int_{a_{i}}^{b_{i}} f d U\right\}\right| \leq \varepsilon$, 
2) $\sum_{i=1}^{r}\left|f\left(x_{i}\right)\left(U\left(b_{i}\right)-U\left(a_{i}\right)\right)-\int_{a_{i}}^{b_{i}} f d U\right| \leq 2 \varepsilon$.

Definition 2.5. Let $F:[a, b] \rightarrow \mathbb{R}$ be any function. Given a system $S$ on a set $A \subseteq[a, b]$ one forms the variational sum $W_{F}(S)=\sum_{i=1}^{r}\left|F\left(b_{i}\right)-F\left(a_{i}\right)\right|$. The $F$-outer measure of the subset $A$ is the number

$$
m_{F}(A)=\inf _{\delta} \sup \left\{W_{F}(S) / S \in \mathcal{S}(A, \delta)\right\},
$$

where $\delta$ runs over all gauges $A \rightarrow \mathbb{R}_{+}$. The following proposition shows that $m_{F}$ is a metric outer measure (for the proof see Proposition 3.3 in [1]).

Proposition 2.6. The functional $m_{F}$ has the following properties:

1) $m_{F}(A) \geq 0$ for every $A \subseteq[a, b]$, and $m_{F}(\emptyset)=0$,

2) $A \subseteq B$ implies $m_{F}(A) \leq m_{F}(B)$,

3) $m_{F}\left(\bigcup_{n=1}^{\infty} A_{n}\right) \leq \sum_{n=1}^{\infty} m_{F}\left(A_{n}\right)$ for every sequence of sets $A_{n} \subseteq[a, b]$,

4) $m_{F}(A \cup B)=m_{F}(A)+m_{F}(B)$ provided $A$ and $B$ are contained in two disjoint open subsets of the interval $[a, b]$.

Remark 2.7. As one could expect, in the special case where $F(x)=x$ the outer measure $m_{F}$ is the Lebesgue outer measure, cf. Proposition 3.4 in [1].

Definition 2.8. Let $U:[a, b] \rightarrow \mathbb{R}$ be a fixed function. One says that a set $A \subseteq[a, b]$ is $U$-null if one can write $A=D \cup N$ with $D$ at most denumerable and $m_{U}(N)=0$. As usual, a property is said to hold $U$-almost everywhere if the exceptional set is $U$-null.

Proposition 2.9. For functions $f, U:[a, b] \rightarrow \mathbb{R}$ the following are equivalent:

1) $f$ is $U$-integrable and $\int_{a}^{x} f d U=0$ for every $x \in(a, b]$,

2) the set $E=\{x \in[a, b] / f(x) \neq 0\}$ satisfies $m_{U}(E)=0$.

Proof. $(1 \Rightarrow 2)$ We show that each set $E_{n}:=\left\{x \in[a, b] /|f(x)| \geq \frac{1}{n}\right\}$ satisfies $m_{U}\left(E_{n}\right)=0$. Given $\varepsilon>0$ there exists a gauge $\delta:[a, b] \rightarrow \mathbb{R}_{+}$such that $|S(f, U, D)|<\varepsilon$ for every $\delta$-fine division $D$ of $[a, b]$. Now let $S$ be any $\delta$-fine system on $E_{n}$. By Saks-Henstock Lemma one obtains

$$
\frac{1}{n} W_{U}(S)=\sum_{i=1}^{r} \frac{1}{n}\left|U\left(b_{i}\right)-U\left(a_{i}\right)\right| \leq \sum_{i=1}^{r}\left|f\left(x_{i}\right)\left(U\left(b_{i}\right)-U\left(a_{i}\right)\right)\right| \leq 2 \varepsilon,
$$

and this proves that $m_{U}\left(E_{n}\right) \leq 2 n \varepsilon$. So the assertion follows. 
$(2 \Rightarrow 1)$ Let $E_{n}:=\{x \in[a, b] / n-1<|f(x)| \leq n\}$. Then there exists for each $n \in \mathbb{N}$ a gauge $\delta_{n}: E_{n} \rightarrow \mathbb{R}_{+}$such that $W_{U}(S)<\varepsilon \frac{1}{n} 2^{-n}$ for any system $S \in \mathcal{S}\left(E_{n}, \delta_{n}\right)$. Taking arbitrary $\delta(x)$ if $f(x)=0$ and $\delta(x)=\delta_{n}(x)$ if $x \in E_{n}$, one gets a gauge $\delta:[a, b] \rightarrow \mathbb{R}_{+}$. For any $\delta$-fine division $D$ of $[a, b]$ one has

$$
|S(f, U, D)| \leq \sum_{n=1}^{\infty} \sum_{x_{i} \in E_{n}} \mid f\left(x_{i}\right)\left(U\left(b_{i}\right)-U\left(a_{i}\right) \mid \leq \sum_{n=1}^{\infty} n W_{U}\left(S_{n}\right)<\varepsilon .\right.
$$

Therefore $f$ is $U$-integrable on $[a, b]$ and $\int_{a}^{b} f \mathrm{~d} U=0$.

\section{Differentiation with Respect to $\mathrm{VBG}^{\mathrm{o}}$ Functions}

Definition 3.1. Let $F, U:[a, b] \rightarrow \mathbb{R}$ be any functions. The lower and upper derivatives of $F$ with respect to $U$,

$$
\underline{\mathrm{D}}_{U} F(x)=\liminf _{y \rightarrow x} \frac{F(y)-F(x)}{U(y)-U(x)} \text { and } \overline{\mathrm{D}}_{U} F(x)=\limsup _{y \rightarrow x} \frac{F(y)-F(x)}{U(y)-U(x)},
$$

are defined for all $x \in[a, b]$ such that $U(y) \neq U(x)$ in a neighborhood of $x$. The function $F$ is differentiable relatively to $U$, or shortly $U$-differentiable, at $x$ if $\underline{\mathrm{D}} F(x)=\overline{\mathrm{D}} F(x) \in \mathbb{R}$, this common value being denoted by $F_{U}^{\prime}(x)$.

We shall use the following version of the Denjoy-Young-Saks theorem:

Theorem 3.2. Let $U:[a, b] \rightarrow \mathbb{R}$ be any strictly increasing function. Then a function $F:[a, b] \rightarrow \mathbb{R}$ is $U$-differentiable at $U$-almost every point of the sets $\left\{x \in[a, b] / \underline{\mathrm{D}}_{U} F(x)>-\infty\right\}$ and $\left\{x \in[a, b] / \overline{\mathrm{D}}_{U} F(x)<\infty\right\}$.

Proof. This is a particular case of Théorème 7 in [2].

Definition 3.3. One says that a function $F:[a, b] \rightarrow \mathbb{R}$ is of bounded variation on a set $E \subseteq[a, b]$, or $V B^{\circ}$ on $E$, if one has $m_{F}(E)<\infty$. One says that the function $F$ is of generalized bounded variation, or $V B G^{\circ}$, if there exists a decomposition $[a, b]=\bigcup_{n=1}^{\infty} E_{n}$ (not necessarily disjoint) such that $F$ is of bounded variation on each subset $E_{n}$.

Remark 3.4. Since a function $F:[a, b] \rightarrow \mathbb{R}$ is continuous at $x$ if and only if $m_{F}(\{x\})=0$, it follows that the set of discontinuities of a $\mathrm{VBG}^{\circ}$ function is at most denumerable.

Lemma 3.5. If a function $F:[a, b] \rightarrow \mathbb{R}$ is of bounded variation on a subset $E \subseteq[a, b]$, then there exist a strictly increasing function $H:[a, b] \rightarrow \mathbb{R}$ and $a$ gauge $\delta: E \rightarrow \mathbb{R}_{+}$such that

$$
x \in E \text { and }|y-x|<\delta(x) \text { imply }|F(y)-F(x)| \leq|H(y)-H(x)| .
$$


Proof. There exists a gauge $\delta: E \rightarrow \mathbb{R}_{+}$such that $W_{F}(S)<m_{F}(E)+1$ for every $\delta$-fine system $S$ on $E$. Then the function

$$
H(x):=x+\sup \left\{W_{F}(S) / S \in \mathcal{S}(E, \delta) \text { and } S \subseteq[a, x]\right\}
$$

satisfies the desired condition (easy verification).

Lemma 3.6. A function $F:[a, b] \rightarrow \mathbb{R}$ is of generalized bounded variation if and only if there exists a strictly increasing function $H:[a, b] \rightarrow \mathbb{R}$ such that

$$
|D|_{H} F(x):=\limsup _{y \rightarrow x}\left|\frac{F(y)-F(x)}{H(y)-H(x)}\right|<\infty \text { for every } x \in[a, b] .
$$

Proof. $(\Rightarrow)$ By definition one has $[a, b]=\bigcup_{n=1}^{\infty} E_{n}$ with $m_{F}\left(E_{n}\right)<\infty$ for every $n \in \mathbb{N}$. Considering for each integer $n$ a function $H_{n}:[a, b] \rightarrow \mathbb{R}$ and a gauge $\delta_{n}: E_{n} \rightarrow \mathbb{R}_{+}$as in the preceding lemma, one defines the function

$$
H(x):=\sum_{n=1}^{\infty} \frac{1}{2^{n}} \cdot \frac{H_{n}(x)-H_{n}(a)}{H_{n}(b)-H_{n}(a)} .
$$

For $x \in E_{n}$ one remarks that $|D|_{H} F(x) \leq 2^{n}\left(H_{n}(b)-H_{n}(a)\right)$.

$(\Leftarrow)$ For each set $E_{n}:=\left\{x \in[a, b] /|D|_{H} F(x)<n\right\}$ one easily proves the inequality $m_{F}\left(E_{n}\right) \leq n(H(b)-H(a))$.

Remark 3.7. According to a theorem of Ward (cf. [9] page 236) it follows that a function $F:[a, b] \rightarrow \mathbb{R}$ is $\mathrm{VBG}^{\circ}$ if and only if it is bounded and $\mathrm{VBG}_{*}$ in the sense of Saks.

Lemma 3.8. Let $H:[a, b] \rightarrow \mathbb{R}$ be a strictly increasing function and let $A$ be a subset of $[a, b]$ with $m_{H}(A)=0$. If the function $F:[a, b] \rightarrow \mathbb{R}$ satisfies $|D|_{H} F(x)<\infty$ for every $x \in A$, then one has $m_{F}(A)=0$.

Proof. We show that $m_{F}\left(A_{n}\right)=0$, where $A_{n}:=\left\{x \in A /|D|_{H} F(x)<n\right\}$. Given $\varepsilon>0$ there exists a gauge $\delta: A_{n} \rightarrow \mathbb{R}_{+}$such that $W_{H}(S)<\varepsilon$ for every system $S \in \mathcal{S}\left(A_{n}, \delta\right)$. We may assume that $x \in A_{n}$ and $|y-x|<\delta(x)$ imply $|F(y)-F(x)|<n|H(y)-H(x)|$. Then $W_{F}(S)<n \varepsilon$ for every $S \in \mathcal{S}\left(A_{n}, \delta\right)$, and this proves that $m_{F}\left(A_{n}\right) \leq n \varepsilon$.

Lemma 3.9. Let $H:[a, b] \rightarrow \mathbb{R}$ be a strictly increasing function. If a function $F:[a, b] \rightarrow \mathbb{R}$ satisfies $F_{H}^{\prime}(x)=0$ for every $x \in A$, then $m_{F}(A)=0$.

Proof. Easy verification (cf. Lemme 5 in [2]). 
Proposition 3.10. Let $F, U:[a, b] \rightarrow \mathbb{R}$ be two $V B G^{\circ}$ functions. Then $F$ is $U$-differentiable at $U$-almost every point of $[a, b]$.

Proof. Let $H_{F}, H_{U}:[a, b] \rightarrow \mathbb{R}$ be strictly increasing functions as in 3.6 and consider the function $H(x):=H_{F}(x)+H_{U}(x)$. By Theorem 3.2 the interval $[a, b]$ can be decomposed into the disjoint union of

1) a set $E_{1}$ where $F$ and $U$ are $H$-differentiable, and

2) a $H$-null set $E_{2}$.

By 3.9 the set $E_{0}=\left\{x \in E_{1} / U_{H}^{\prime}(x)=0\right\}$ is $U$-null, and by 3.8 the set $E_{2}$ is $U$-null. Now if $x \in E_{1} \backslash E_{0}$, then one has $F_{U}^{\prime}(x)=F_{H}^{\prime}(x) \cdot U_{H}^{\prime}(x)^{-1}$.

\section{The Fundamental Theorem}

Throughout this section $U:[a, b] \rightarrow \mathbb{R}$ is a fixed continuous $\mathrm{VBG}^{\circ}$ function.

Definition 4.1. A function $F:[a, b] \rightarrow \mathbb{R}$ is called $U$-Lipschitzian on a set $E \subseteq[a, b]$, or $L Z_{U}$ on $E$, if there exists $C>0$ such that $m_{F}(A) \leq C \cdot m_{U}(A)$ for every subset $A \subseteq E$. The function $F$ is called generalized $U$-Lipschitzian, or $L Z G_{U}$, if there exists some decomposition $[a, b]=\bigcup_{n=1}^{\infty} E_{n}$ such that $F$ is $U$-Lipschitzian on each subset $E_{n}$.

Similarly, a function $F:[a, b] \rightarrow \mathbb{R}$ is called $U$-absolutely continuous on a set $E$, or $A C_{U}$ on $E$, if for any $\varepsilon>0$ there exists $\delta>0$ such that $A \subseteq E$ and $m_{U}(A)<\delta$ imply $m_{F}(A)<\varepsilon$. And it is called generalized $U$-absolutely continuous, or $A C G_{U}$, if there exists some decomposition $[a, b]=\bigcup_{n=1}^{\infty} E_{n}$ such that $F$ is $U$-absolutely continuous on each subset $E_{n}$.

Finally, one says that a function $F:[a, b] \rightarrow \mathbb{R}$ is $U$-variationally normal, or shortly $U$-normal, if $m_{U}(A)=0$ implies $m_{F}(A)=0$.

Lemma 4.2. If the function $U$ is of bounded variation on the set $E \subseteq[a, b]$, then the function $V(x)=m_{U}(E \cap[a, x])$ is continuous.

Proof. Since $U$ is continuous one has $m_{U}(E \cap[c, d])=m_{U}(E \cap(c, d))$ for every subinterval $[c, d] \subseteq[a, b]$. Now let $x_{n}$ be a strictly increasing sequence with $x_{0}=a$ and $\lim x_{n}=x$. We show that $V\left(x_{n}\right)$ converges to $V(x)$. Using the subadditivity of $m_{U}$, cf. Proposition 2.6, one obtains

$$
V(x) \leq \sum_{n=1}^{\infty} m_{U}\left(E \cap\left[x_{n-1}, x_{n}\right)\right)=\sum_{n=1}^{\infty} m_{U}\left(E \cap\left(x_{n-1}, x_{n}\right)\right) .
$$

And using Proposition 2.6 once again one concludes that

$$
\sum_{n=1}^{s} m_{U}\left(E \cap\left(x_{n-1}, x_{n}\right)\right)=m_{U}\left(E \cap \bigcup_{n=1}^{s}\left(x_{n-1}, x_{n}\right)\right)=V\left(x_{s}\right)
$$


for every $s \in \mathbb{N}$. Thus $V(x) \leq \lim V\left(x_{s}\right) \leq V(x)$ and the assertion is proved. The continuity on the right side of $x$ is proved similarly, by considering the function $V(b)-V(x)=m_{U}(E \cap(x, b])$.

Lemma 4.3. Any $L Z G_{U}$ function is $A C G_{U}$, and any $A C G_{U}$ function is $V B G^{\circ}$ and $U$-variationally normal.

Proof. We show that if $F:[a, b] \rightarrow \mathbb{R}$ is $\mathrm{AC}_{U}$ and the function $U$ is $\mathrm{VB}^{\circ}$ on a set $E \subseteq[a, b]$, then $F$ is $\mathrm{VB}^{\circ}$ on $E$ (the other affirmations are evident). We consider the function $V(x)$ of the preceding lemma. By definition there exists $\delta>0$ such that $A \subseteq E$ and $m_{U}(A)<\delta$ imply $m_{F}(A)<1$. And by continuity of the function $V$ we can choose a partition $a=x_{0}<x_{1}<\ldots<x_{n}=b$ such that $V\left(x_{i}\right)-V\left(x_{i-1}\right)=m_{U}\left(E \cap\left[x_{i-1}, x_{i}\right]\right)<\delta$ for every $i=1, \ldots, n$. Thus we obtain $m_{F}(E)<n$, and the assertion is proved.

Proposition 4.4. Let $f:[a, b] \rightarrow \mathbb{R}$ be integrable relatively to $U$. Then the indefinite integral $F(x)=\int_{a}^{x} f \mathrm{~d} U$ is $L Z G_{U}$.

Proof. We show that if the function $U$ is $\mathrm{VB}^{\circ}$ on the set $E \subseteq[a, b]$, then $F$ is $\mathrm{LZ}_{U}$ on each subset $E_{n}:=\{x \in E /|f(x)| \leq n\}$. So let $A \subseteq E_{n}$ be a fixed subset. Given $\varepsilon>0$ there exist two gauges $\delta_{1}$ on $[a, b]$ and $\delta_{2}$ on $A$ such that

1) $\left|S(f, U, D)-\int_{a}^{b} f \mathrm{~d} U\right|<\varepsilon$ for every $\delta_{1}$-fine division $D$ of $[a, b]$,

2) $W_{U}(S)<m_{U}(A)+\varepsilon$ for every system $S \in \mathcal{S}\left(A, \delta_{2}\right)$.

We consider the gauge $\delta: A \rightarrow \mathbb{R}_{+}$defined by $\delta(x)=\min \left(\delta_{1}(x), \delta_{2}(x)\right)$. Now let $S$ be any $\delta$-fine system on $A$. By Saks-Henstock Lemma we have

$$
\begin{gathered}
W_{F}(S)=\sum_{i=1}^{r}\left|F\left(b_{i}\right)-F\left(a_{i}\right)\right| \leq \sum_{i=1}^{r}\left|f\left(x_{i}\right)\left(U\left(b_{i}\right)-U\left(a_{i}\right)\right)\right|+ \\
\sum_{i=1}^{r}\left|F\left(b_{i}\right)-F\left(a_{i}\right)-f\left(x_{i}\right)\left(U\left(b_{i}\right)-U\left(a_{i}\right)\right)\right| \leq n\left(m_{U}(A)+\varepsilon\right)+2 \varepsilon .
\end{gathered}
$$

Thus we obtain $m_{F}(A) \leq n \cdot m_{U}(A)+(n+2) \varepsilon$, and since $\varepsilon$ is arbitrary this proves that $F$ is $U$-Lipschitzian on the set $E_{n}$.

For the next proposition it is useful to introduce some notations. Given a function $F$ on $[a, b]$ we put $E_{F}=\{x \in[a, b] / F$ is not $U$-differentiable at $x\}$, and we define the derivative $D_{U} F:[a, b] \rightarrow \mathbb{R}$ by $D_{U} F(x)=F_{U}^{\prime}(x)$ if $x \notin E_{F}$ and $D_{U} F(x)=0$ if $x \in E_{F}$.

Proposition 4.5. Let $F:[a, b] \rightarrow \mathbb{R}$ be a function such that $m_{F}\left(E_{F}\right)=0$. Then the derivative $D_{U} F$ is integrable relatively to $U$. Furthermore, one has $\int_{a}^{x} D_{U} f \mathrm{~d} U=F(x)-F(a)$ for every $x \in(a, b]$. 
Proof. We show that $\int_{a}^{b} D_{U} f \mathrm{~d} U=F(b)-F(a)$. Let $[a, b]=\bigcup_{n=1}^{\infty} E_{n}$ be a disjoint decomposition such that $m_{U}\left(E_{n}\right)<\infty$ for every $n \in \mathbb{N}$. There exists for each $n$ a gauge $\delta_{n}^{\prime}: E_{n} \rightarrow \mathbb{R}_{+}$such that $W_{U}\left(S_{n}\right)<m_{U}\left(E_{n}\right)+1$ for every system $S_{n} \in \mathcal{S}\left(E_{n}, \delta_{n}^{\prime}\right)$. Define $\varepsilon_{n}>0$ by $2^{n} \varepsilon_{n}\left(m_{U}\left(E_{n}\right)+1\right)=\varepsilon$. For each $x \in E_{n} \backslash E_{F}$ there exists $\delta_{n}(x)>0$ such that $|y-x|<\delta_{n}(x)$ implies

$$
\left|F(y)-F(x)-F_{U}^{\prime}(x)(U(y)-U(x))\right| \leq \varepsilon_{n}|U(y)-U(x)| .
$$

One may assume that $\delta_{n}(x) \leq \delta_{n}^{\prime}(x)$. And by hypothesis there exists a gauge $\delta: E_{F} \rightarrow \mathbb{R}_{+}$such that $W_{F}(S)<\varepsilon$ for every system $S \in \mathcal{S}\left(E_{F}, \delta\right)$. One thus gets a gauge $\delta:[a, b] \rightarrow \mathbb{R}_{+}$. Now let $D$ be any $\delta$-fine division of the interval $[a, b]$. Then one has the following inequality:

$$
\begin{aligned}
& \left|S\left(D_{U} F, U, D\right)-F(b)+F(a)\right| \leq \sum_{x_{i} \in E_{F}}\left|F\left(b_{i}\right)-F\left(a_{i}\right)\right|+ \\
& \sum_{n=1}^{\infty} \sum_{x_{i} \in E_{n} \backslash E_{F}}\left|F_{U}^{\prime}\left(x_{i}\right)\left(U\left(b_{i}\right)-U\left(x_{i}\right)\right)-\left(F\left(b_{i}\right)-F\left(x_{i}\right)\right)\right|+ \\
& \sum_{n=1}^{\infty} \sum_{x_{i} \in E_{n} \backslash E_{F}}\left|F_{U}^{\prime}\left(x_{i}\right)\left(U\left(x_{i}\right)-U\left(a_{i}\right)\right)-\left(F\left(x_{i}\right)-F\left(a_{i}\right)\right)\right|< \\
& \varepsilon+\sum_{n=1}^{\infty} \varepsilon_{n} W_{U}\left(S_{n}^{+}\right)+\sum_{n=1}^{\infty} \varepsilon_{n} W_{U}\left(S_{n}^{-}\right) \leq \varepsilon+2 \sum_{n=1}^{\infty} 2^{-n} \varepsilon=3 \varepsilon,
\end{aligned}
$$

and this proves that $D_{U} F$ is integrable with respect to $U$.

Corollary 4.6. Let $F:[a, b] \rightarrow \mathbb{R}$ be a continuous function. If there exists a denumerable set $D \subseteq[a, b]$ such that $F$ is $U$-differentiable on $[a, b] \backslash D$, then $F(x)=F(a)+\int_{a}^{x} D_{U} F \mathrm{~d} U$ for every $x \in(a, b]$.

Proof. This is immediate since $m_{F}(D)=0$, cf. Remark 3.4.

Theorem 4.7. For a function $F:[a, b] \rightarrow \mathbb{R}$ the following are equivalent:

1) $F$ is an indefinite integral relatively to $U$,

2) $F$ is $L Z G_{U}$,

3) $F$ is $A C G_{U}$,

4) $F$ is $V B G^{\circ}$ and $U$-normal,

5) $F$ is $U$-differentiable $U$-almost everywhere and $U$-normal.

Proof. This follows from Propositions 4.4, 4.3, 3.10 and 4.5 (another equivalent condition will be given in Corollary 5.6).

Corollary 4.8. Let $f:[a, b] \rightarrow \mathbb{R}$ be a $U$-integrable function and let $F(x)=$ $\int_{a}^{x} f \mathrm{~d} U$ be its indefinite integral. Then $F_{U}^{\prime}(x)=f(x) U$-almost everywhere.

Proof. By 2.9 the set $\left\{x \in[a, b] / f(x) \neq D_{U} F(x)\right\}$ is $U$-null. 


\section{The Lusin Condition (N)}

Let $F:[a, b] \rightarrow \mathbb{R}$ be fixed. We want to compare the two following conditions (where $m$ denotes the Lebesgue outer measure):

1) $m(A)=0$ implies $m_{F}(A)=0$ (see Definition 4.1), and

2) $m(A)=0$ implies $m(F(A))=0$, i.e. the Lusin condition $(\mathrm{N})$.

Lemma 5.1. For any set $A \subseteq[a, b]$ with $m_{F}(A)=0$ one has $m(F(A))=0$.

Proof. Given $\varepsilon>0$ there exists a gauge $\delta: A \rightarrow \mathbb{R}_{+}$such that $W_{F}(S)<\varepsilon$ for every system $S \in \mathcal{S}(A, \delta)$. By the so-called Covering Lemma (McLeod [7] page 143) there exist two (possibly finite) sequences of non-overlapping intervals $I_{n}=\left[a_{n}, b_{n}\right]$ and of points $x_{n} \in I_{n} \cap A$ such that

$$
I_{n} \subseteq\left(x_{n}-\delta\left(x_{n}\right), x_{n}+\delta\left(x_{n}\right)\right) \text { for every } n \text {, and } A \subseteq \bigcup_{n} I_{n} .
$$

For each $n$ we define $m_{n}=\inf \left(F, I_{n}\right)$ and $M_{n}=\sup \left(F, I_{n}\right)$, and we choose a point $y_{n} \in I_{n}$ with $M_{n}-m_{n} \leq 3\left|F\left(y_{n}\right)-F\left(x_{n}\right)\right|$. For every finite sum one has $\sum_{n=1}^{r}\left(M_{n}-m_{n}\right) \leq 3 W_{F}\left(S_{r}\right)<3 \varepsilon$. Therefore $\sum_{n}\left(M_{n}-m_{n}\right) \leq 3 \varepsilon$, and this shows that $m(F(A)) \leq 3 \varepsilon$ since $F(A) \subseteq \bigcup_{n}\left[m_{n}, M_{n}\right]$.

Lemma 5.2. Let $C_{F}=\{x \in[a, b] / y \leq x \leq z$ implies $F(y) \leq F(x) \leq F(z)\}$. If the function $F$ is continuous on a subset $A \subseteq C_{F}$ satisfying $m(F(A))=0$, then one has $m_{F}(A)=0$.

Proof. Given $\varepsilon>0$ there exists by hypothesis a gauge $\eta: F(A) \rightarrow \mathbb{R}_{+}$such that $W_{\text {id }}(T)<\varepsilon$ for every system $T \in \mathcal{S}(F(A), \eta$ ), cf. Remark 2.7 (one may also work with the usual definition of sets of measure zero). By continuity of $F$ there exists a gauge $\delta: A \rightarrow \mathbb{R}_{+}$such that $x \in A$ and $|y-x|<\delta(x)$ imply $|F(y)-F(x)|<\eta(F(x))$. If $S$ is any $\delta$-fine system on $A$, then one has

$$
W_{F}(S)=\sum_{i=1}^{r}\left(F\left(b_{i}\right)-F\left(x_{i}\right)+F\left(x_{i}\right)-F\left(a_{i}\right)\right)=W_{\mathrm{id}}\left(T_{1}\right)+W_{\mathrm{id}}\left(T_{2}\right)
$$

(use that $x_{i} \in C_{F}$ for every $1 \leq i \leq r$ ), and therefore $m_{F}(A) \leq 2 \varepsilon$.

Proposition 5.3. For a subset $A \subseteq[a, b]$ the following are equivalent:

1) $m_{F}(A)=0$

2) $F$ is continuous on $A, m(F(A))=0$ and $m_{F}(A)<\infty$. 
Proof. $(2 \Rightarrow 1)$ Since $F$ is of bounded variation on $A$ there exists by 3.5 a strictly increasing function $H:[a, b] \rightarrow \mathbb{R}$ and a gauge $\delta: A \rightarrow \mathbb{R}_{+}$such that $x \in A$ and $|y-x|<\delta(x)$ imply $|F(y)-F(x)| \leq|H(y)-H(x)|$. We remark that this implies $m_{F}(N)=0$ for every subset $N \subseteq A$ satisfying $m_{H}(N)=0$. Since by Theorem 3.2 the set $E=\{x \in A / F$ is not $H$-differentiable at $x\}$ is $H$-null we deduce that $m_{F}(E) \leq m_{F}(N)+m_{F}(D)=0$.

By Lemma 3.9 the set $A_{0}=\left\{x \in A / F_{H}^{\prime}(x)=0\right\}$ satisfies $m_{F}(A)=0$. So it remains to consider the sets $A_{ \pm}=\left\{x \in A / \pm F_{H}^{\prime}(x)>0\right\}$. Obviously, one has $A_{+} \subseteq \bigcup_{n=1}^{\infty} A_{n}$, where

$$
A_{n}=\left\{x \in A / x \in[y, z] \subseteq\left(x-\frac{1}{n}, x+\frac{1}{n}\right) \Rightarrow F(y) \leq F(x) \leq F(z)\right\} .
$$

By the preceding lemma one obtains $m_{F}\left(A_{n}\right)=0$ for every $n \in \mathbb{N}$ (divide the interval $[a, b]$ into finitely many small intervals). Therefore $m_{F}\left(A_{+}\right)=0$, and similarly $m_{F}\left(A_{-}\right)=0$, which proves the proposition.

Question 5.4. The example of Saks ([9] p. 224) shows that the hypothesis $m_{F}(A)<\infty$ cannot be released. But could one put in place of it the weaker assumption that $F$ is differentiable almost everywhere? Or in other words, is there any function satisfying the Lusin condition $(N)$ that is continuous and differentiable almost everywhere when not $V B G^{\circ}$ ?

Definition 5.5. Let $U:[a, b] \rightarrow \mathbb{R}$ be a continuous $\mathrm{VBG}^{\circ}$ function as in the preceding section. One says that a function $F:[a, b] \rightarrow \mathbb{R}$ satisfies the Lusin condition $U-(N)$ if $m(U(A))=0$ implies $m(F(A))=0$.

Corollary 5.6. For a function $F:[a, b] \rightarrow \mathbb{R}$ the following are equivalent;

1) $F$ is an indefinite integral with respect to $U$,

6) $F$ is continuous, $V B G^{\circ}$ and it satisfies the Lusin condition $U-(N)$.

Proof. Using Proposition 5.3 one obtains $m(U(A))=0$ iff $m_{U}(A)=0$, and similarly $m(F(A))=0$ iff $m_{F}(A)=0$.

As another corollary of Proposition 5.3 we give the following substitution theorem for the Kurzweil-Henstock integral (which might be proved also by a more direct method):

Corollary 5.7. Let $U:[a, b] \rightarrow \mathbb{R}$ be continuous and $V B G^{\circ}$, and consider the interval $[c, d]=U([a, b])$. If the function $f:[c, d] \rightarrow \mathbb{R}$ is measurable and bounded, then $f \circ U$ is integrable relatively to $U$ and $\int_{a}^{b} f \circ U \mathrm{~d} U=\int_{U(a)}^{U(b)} f$. 
Proof. Let $F(y)=\int_{c}^{y} f$ be the indefinite integral of $f$. Clearly, the function $F$ is Lipschitzian, and this implies that $F \circ U$ is $\mathrm{LZG}_{U}$. By the fundamental theorem 4.7 we obtain $\int_{U(a)}^{U(b)} f=F(U(b))-F(U(a))=\int_{a}^{b} D_{U}(F \circ U) \mathrm{d} U$. So we are led to consider the following sets:

1) $A=\{x \in[a, b] / F \circ U$ is not $U$-differentiable at $x\}$,

2) $B=\left\{x \notin A /(F \circ U)_{U}^{\prime}(x) \neq f(U(x))\right\}$,

3) $C=\left\{y \in[c, d] / F\right.$ is not differentiable at $y$ or $\left.F^{\prime}(y) \neq f(y)\right\}$,

One has $m_{U}(A)=0$ by Theorem 3.10 (use that $U$ is continuous). And since $U(B) \subseteq C$ is of measure zero one gets $m_{U}(B)=0$ by Proposition 5.3. Hence the set $E=\left\{x \in[a, b] / D_{U}(F \circ U)(x) \neq f(U(x))\right\}$ satisfies $m_{U}(E)=0$, and the assertion follows from Proposition 2.9.

In particular, if $U$ is an indefinite integral, i.e. $U(x)=U(a)+\int_{a}^{x} g$, then $(f \circ U) \cdot g$ is integrable and $\int_{a}^{b}(f \circ U) \cdot g=\int_{U(a)}^{U(b)} f$ (left as an exercise).

\section{References}

[1] C.-A. Faure, A descriptive definition of some multidimensional gauge integrals, Czech. Math. J. 45 (1995), 549-562.

[2] C.-A. Faure, Sur le théorème de Denjoy-Young-Saks, C. R. Acad. Sci. Paris Série I Math. 320 (1995), 415-418.

[3] J. Jarník and J. Kurzweil, A general form of the product integral and linear ordinary differential equations, Czech. Math. J. 37 (1987), 642659 .

[4] W. B. Jurkat and R. W. Knizia, A characterization of multi-dimensional Perron integrals and the fundamental theorem, Can. J. Math. 43 (1991), $526-539$.

[5] W. B. Jurkat and R. W. Knizia, Generalized absolutely continuous interval functions and multi-dimensional Perron integration, Analysis $\mathbf{1 2}$ (1992), 303-313.

[6] J. Mawhin, Generalized multiple Perron integrals and the Green-Goursat theorem for differentiable vector fields, Czech. Math. J. 31 (1981), 614632 . 
[7] R. M. McLeod, The generalized Riemann integral, Mathematical Association of America, Washington D.C., 1980.

[8] W. F. Pfeffer, The divergence theorem, Trans. Amer. Math. Soc. 295 (1986), 665-685.

[9] S. Saks, Theory of the integral, Dover, New York, 1964.

[10] Á. Száz, The fundamental theorem of calculus in an abstract setting, Tatra Mt. Math. Publ. 2 (1992), 167-174. 